\title{
NOTE ON THE RELATION BETWEEN COMPOUND AND COMPOSED POISSON PROCESSES \\ Carl Philipson
}

I. In an article on such processes in ASTIN Bull. (II-3, pp. 450-45I) the author has derived a relation between cPp i.n.s. (compound Poisson processes in the narrow sense) and composed Poisson processes, which is quite general. In this note this relation shall be simplified under the rather weak condition that $P_{0}(t)$, $P_{n}(t)$ being the distribution of the number of changes occurring in the interval $(0, t)$, for every fixed value of $t$, tends to a positive limit less than unity when $t$ tends to infinity.

2. By the theory expounded by Thyrion (Bull. de l'Assoc. Act. Belges, I959 and 1963) the characteristic function of the number of events in the interval $(0, t)$ can for each fixed value of $t$ in this case be written

$$
\exp \left[-\theta(t)\left(\frac{-\mathrm{I}}{\theta(t)} \sum_{\nu=1}^{\infty} q_{\nu}(t) e^{i n v}-\mathrm{I}\right)\right],
$$

where $\theta(t)=\log P_{0}(t), q_{v}(t)=(-t)^{\nu} \theta((v) t) / \nu !, \sum_{\nu=2}^{\infty} q_{v}(t)=-\theta(t)$, $\eta$ is a real variable and $i$ the imaginary unity.

It is easily seen that ( $\mathrm{I}$ ) is equal to the following expression

$$
\exp \left[\sum_{v=1}^{\infty} q_{v}(t)\left(e^{i n v}-\mathrm{I}\right)\right]
$$

which has the form of a characteristic function of the number of changes in a composed Poisson process.

3. The original cPp i.n.s. is, generally, heterogeneous in both time and space, i.e. the intensity function $p_{n}(t)$ depends on both $n$ and $t$. A transformation of the time parameter leads to an intensity function $p_{n}\left(t^{*}\right)$, which for the Polya process is independent of $t^{*}$, i.e. the transformed Polya process is homogeneous in time but, still, heterogeneous in space. Except for the Poisson process 
where the intensity function does not depend on $n$, the intensity function $\bar{p}_{n}\left(t^{*}\right)$ depends for other cPp i.n.s. on $n$. Consequently, as was stated in the paper in ASTIN Bull. quoted above, the processes defined by (2) are not particular cases of the infinitely divisible distributions. 